\title{
A 32*32 PARYlene-Pyrolyzed Carbon Bolometer IMAGER
}

\author{
Matthieu Liger, Yu-Chong Tai \\ California Institute of Technology, Pasadena California, USA
}

\begin{abstract}
Here we present a novel and simple way to fabricate uncooled infrared detectors suitable for integration into large-area arrays. The design is based on thin-film carbon obtained by means of parylene pyrolysis. We demonstrate the first micromachined carbon uncooled bolometer array made of two layers of self-supporting pyrolyzed-parylene carbon having different process-tuned properties. Measurements show that the target temperature resolution, or NETD (Noise-Equivalent Temperature Difference) to target temperature can be as low as $35 \mathrm{mK}$ for $100 \mu$ s electrical signal integration time, respectively. This matches the current state of the art which is very promising considering the fact that this is the first time pyrolytic carbon has been used to fabricate a microbolometer array.
\end{abstract}

\section{INTRODUCTION}

Most of the radiation emitted by bodies around room temperature lies in the Long-Wavelength Infrared (LWIR) region, with the maximum emission occurring at a wavelength close to $10 \mu \mathrm{m}$. Therefore, in order to remotely measure the temperature of objects around room temperature, a sensor must be able to detect photons of such wavelength. While semiconductor photon detectors can be used for this purpose, these detectors must be cryogenically cooled, due to the small bandgap requirement. Another kind of detectors called thermal detectors indirectly measure LWIR radiation by absorbing the emitted photons. The induced change in temperature can then be measured using a variety of temperature-dependent physical phenomena including pyroelectricity, thermal expansion, thermoelectricity. Resistive bolometer measure the radiationinduced temperature with materials having a temperaturedependent resistivity.

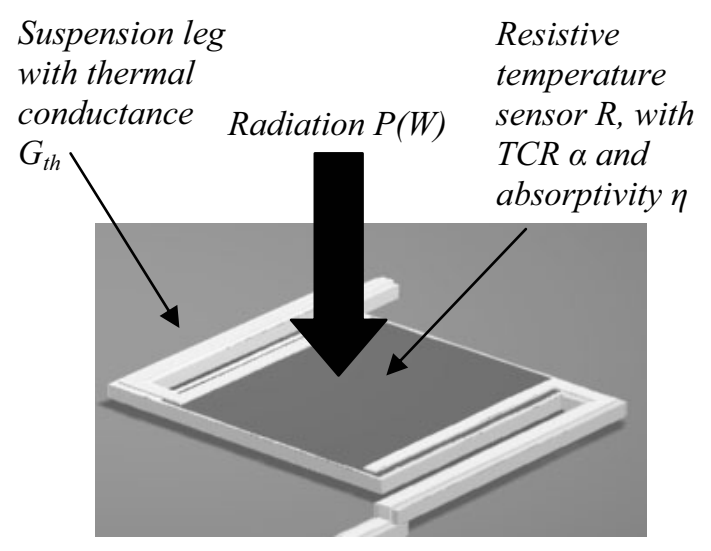

Figure 1: Typical Bolometer
Figure 1 shows the standard structure of a resistive bolometer: a free-standing temperature-sensitive element anchored to a substrate by low thermal-conductance suspension beams. Equations 1 and 2 show the expression of the responsivity and NETD respectively. $\mathrm{P}$ is the incident power, $\Delta \mathrm{V}$ is the voltage change across the bolometer due to $\mathrm{P}, \alpha$ is the temperature coefficient of resistance (TCR), $\eta$ is the pixel absorptivity and $\mathrm{G}_{\mathrm{th}}$ is the thermal conductance to substrate. $\mathrm{F}$ is the F number of the optical system used, $\mathrm{V}_{\mathrm{n}}$ is the bolometer total noise, $A_{d}$ is the bolometer area and $(\mathrm{dM} / \mathrm{dT}) 8 \mu \mathrm{m}-14 \mu \mathrm{m}$ is the change in blackbody excitance in the $8 \mu \mathrm{m}-14 \mu \mathrm{m}$ band for per degree of change on its surface $(\mathrm{dM} / \mathrm{dT})$ is the change in a blackbody's excitance upon temperature change and $\mathrm{F}$, the optics $\mathrm{F}$ number. The key parameters for a bolometer are high TCR, low thermal conductance and low noise.

$$
\begin{gathered}
\mathfrak{R}\left(V . W^{-1}\right)=\frac{\Delta V}{P}=\frac{\alpha \eta V_{\text {bias }}}{G_{\text {th }}} \\
\operatorname{NETD}(K)=\frac{4 F^{2} V_{n}}{\mathfrak{R} A_{d}\left(\frac{d M}{d T}\right)_{8 \mu m-14 \mu m}}
\end{gathered}
$$

Most uncooled bolometers use Vanadium Oxide (VOx) $[1,8]$, amorphous silicon [2] or metals as temperature-sensitive material. The suspension beams are usually made of silicon nitride or polysilicon. In our previous work [6], we demonstrated that the pyrolysis of parylene can be used to produce carbon with various resistivities. In [7], we presented a single-element carbon bolometer made of two layer of pyrolytic carbon obtained through parylene pyrolysis. Here, a new $32 \times 32$ carbon bolometer imager is presented with IR images and the first noise characterization for pyrolyzed-parylene carbon.

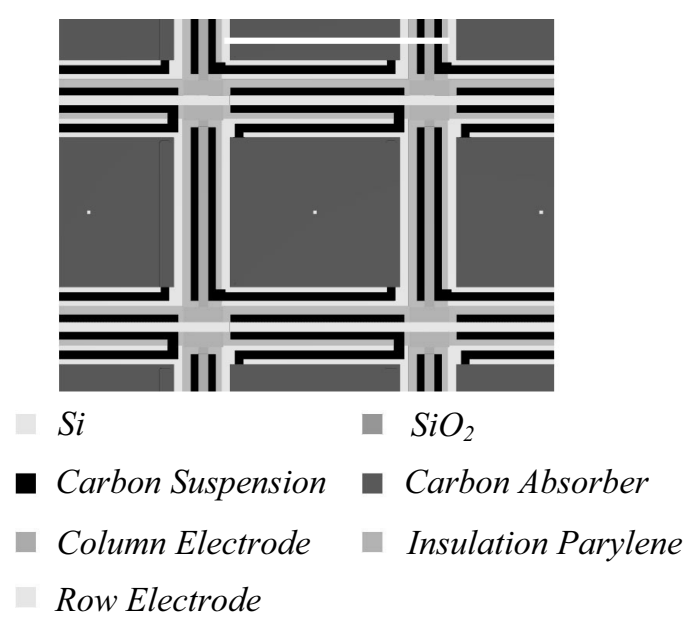

Figure 2: Pixel Layout 


\section{DESIGN}

Figure 2 shows the layout of a single carbon bolometer pixel. The main difference between this bolometer pixel and the "conventional" pixel is the fact that both the absorber region and the suspension legs are made of carbon obtained by pyrolysis of parylene. The absorber area is made of silicon having a high resistivity and TCR. On the other hand, the suspension legs are made of carbon also obtained through parylene pyrolysis but having low resistivity and lower TCR. The different values resistivities and TCRs are achieved by performing the pyrolysis at different temperatures. The pitch is $75 \mu \mathrm{m}$ in both directions and the absorber dimensions are $50 \mu \mathrm{m} * 50 \mu \mathrm{m}$. The fill factor is therefore $44.4 \%$. The suspensions are $60 \mu \mathrm{m}$ in length and $2 \mu \mathrm{m}$ by width. Using a pyrolysis temperature of $800^{\circ} \mathrm{C}$ and $640^{\circ}$ for the suspension and absorber area respectively, we can make sure that the total electrical resistance of each pixel is dominated by the absorber area, while the achieving a high TCR where the radiation-induced temperature-change is the highest [7]. Row access and column access metal electrodes are located on the sides of the pixels and are supported by the bulk silicon and the bolometers are to be released in TMAH. Figure 3 shows the bolometer cross-section.

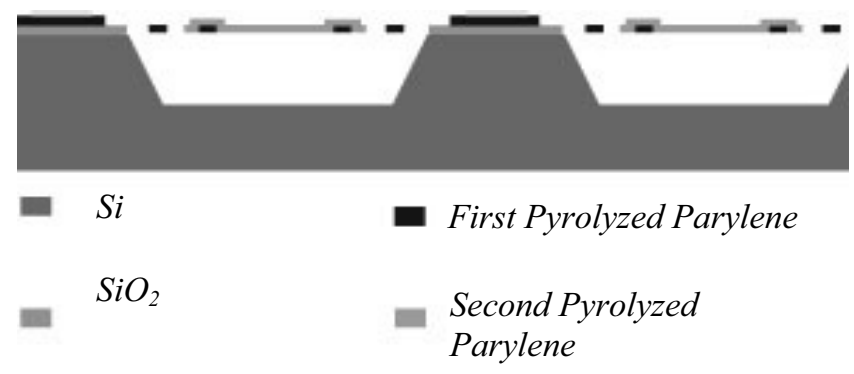

$\mathrm{Cr} / \mathrm{Au}$

Figure 3: Bolometer Array Cross-section

\section{FABRICATION}

The fabrication begins with $4000 \AA$ oxide. The oxide is patterned as a mask for the final TMAH release. A first layer of parylene $(4 \mu \mathrm{m})$ is deposited and pyrolyzed to carbon at $800^{\circ} \mathrm{C}$ in nitrogen. The carbon is then etched by an $\mathrm{O} 2$ plasma in an RIE reactor to define the bolometer suspension beams. The etching rate for this layer of carbon was $0.21 \mu \mathrm{m} / \mathrm{min}$ while the etching rate of photoresist was $0.39 \mu \mathrm{m} / \mathrm{min}$. Surface cleanliness is critical to get good adhesion between pyrolyzed-parylene carbon and silicon and silicon dioxide. Therefore, the wafers are thoroughly cleaned using RCA-1 prior to parylene deposition. Good adhesion is especially critical for the final TMAH release.

A second layer of parylene $(1.6 \mu \mathrm{m})$ is deposited, pyrolyzed at $640^{\circ} \mathrm{C}$ and patterned to create the absorber. A layer of $\mathrm{Cr} / \mathrm{Au} / \mathrm{Cr}(50 \AA / 1000 \AA / 50 \AA)$ is deposited using lift-off to define the row electrodes. Since these electrodes are very long and only $3 \mu \mathrm{m}$ wide, a layer of lift-off photoresist (LOR $3 \mathrm{~B}$ from Microchem) is spun and baked on the wafers prior to the conventional photoresist spin and bake. The wafers are then slightly over-developed in order to create an undercut underneath the photoresist pattern. After the metal evaporation, the wafers are placed in ST22 photoresist stripper at $90^{\circ} \mathrm{C}$ for 10 hours to complete the lift-off.

After applying an A-174 silane coating as adhesion promoter on the wafers, a thin layer of parylene $C(0.3 \mu \mathrm{m})$ is deposited and patterned to serve as insulation between the column metal layer and the row metal layer. A layer of $\mathrm{Cr} / \mathrm{Au}$ $(50 \AA / 2000 \AA)$ is then deposited using the lift-off process described above in order to define the column electrodes. The bolometer array is finally undercut in TMAH and dryfreed in a supercritical $\mathrm{CO}_{2}$ dryer to avoid stiction. Figure 4 shows a bolometer pixel before release, and Figure 5 shows a group of released bolometer pixels.

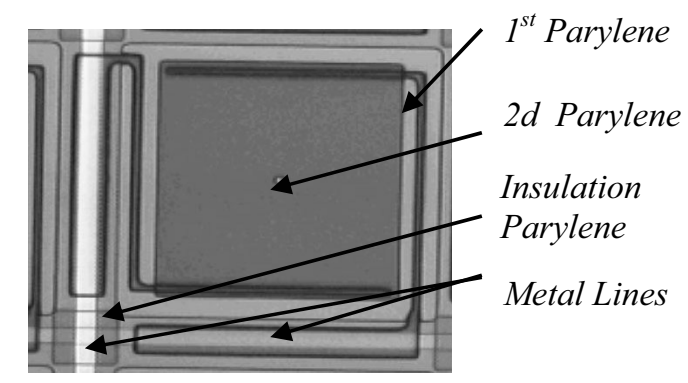

Figure 4: Fabricated Bolometer Pixel

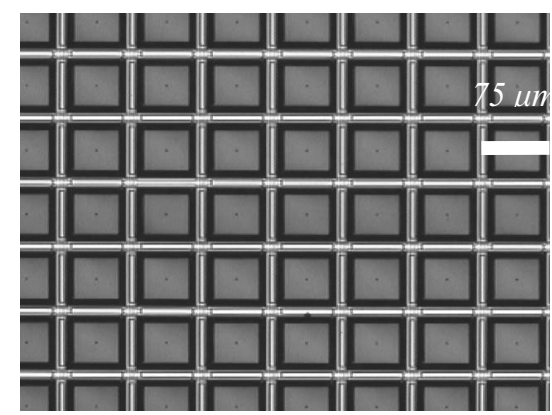

Figure 5: Released Bolometer Pixels

\section{RESULTS}

The average bolometer pixel resistance was measured to be $3.8 \mathrm{M} \Omega$ with a standard deviation of $421 \mathrm{k} \Omega$. This corresponds to an average resistivity of the first layer of pyrolyzed-parylene carbon was measured to be $2.7^{*} 10^{-2} \Omega . \mathrm{cm}$ while the resistivity of the second layer of pyrolyzed-parylene was $190^{*} 10^{2} \Omega . \mathrm{cm}$. This ratio ensures that the total bolometer is indeed dominated by the absorber area. The temperature dependence of the bolometer's resistance was measured in a vacuum oven using a precision thermocouple. As expected, the conductivity of the pyrolyzed-parylene carbon followed an Arrhenius dependence [7]. The TCR was found to be $\alpha$ $=-2.1 \% \mathrm{~K}^{-1}$.

After measuring the TCR of the bolometers, the arrays were placed in a vacuum chamber with electrical feed-throughs and a $\mathrm{ZnS}$ infrared viewport (obtained from ISP Optics). The ZnS window has a transmittance higher than $60 \%$ from $0.5 \mu \mathrm{m}$ to $14 \mu \mathrm{m}$. The I-V curve of the carbon bolometers was measured at $20 \mathrm{mTorr}$ in order to extract their thermal conductance. Figure 6 shows 
the resistance as a function of the input power. The thermal conductance extracted from this curve using the previously measured TCR was $\mathrm{G}_{\mathrm{th}}=6.1 * 10^{-8} \mathrm{~W} \cdot \mathrm{K}^{-1}$.

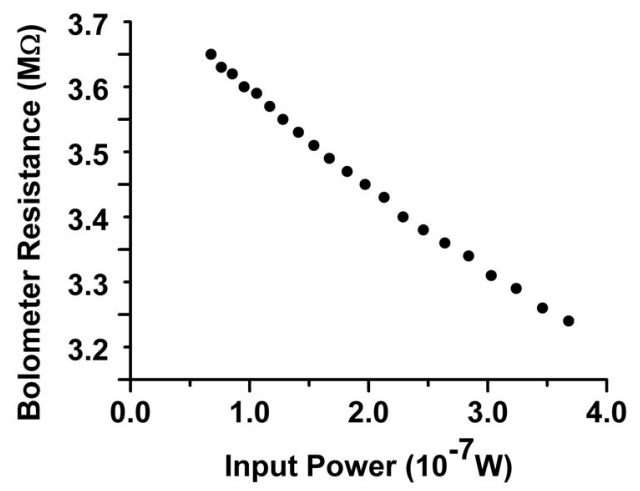

Figure 6: Bolometer Electrothermal Behavior

Then, the time constant of the bolometers was measured using a light chopper wheel to modulate the frequency of the optical input to the bolometer. A spectrum analyzer was used to measure the amplitude of the fundamental component as a function of chopping frequency. As expected the dynamic behavior of the bolometers in vacuum followed that of a first-order system, and the time constant was $31 \mathrm{~ms}$. This is a little too slow for video applications, but acceptable. Using the measured time constant and the calculated thermal conductance, the thermal capacitance is estimated to be $1.9 * 10^{-9} \mathrm{~J} . \mathrm{K}^{-1}$. The absorptivity was estimated using an infrared source and a calibrated pyroelectric sensor (Coherent P1-31). The low-frequency responsivity of the carbon bolometers was compared to the known responsivity of the calibrated commercial detector. The bolometers the absorptivity was then calculated to be $\eta=55 \%$.

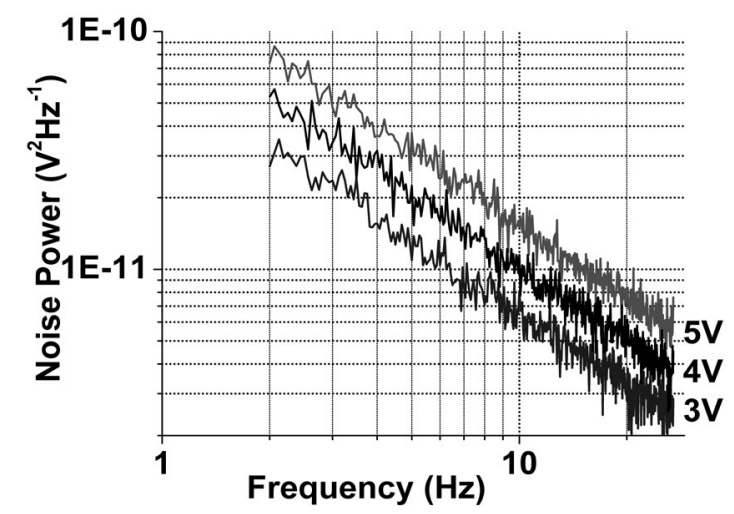

Figure 7: Bolometer Low Frequency Noise Spectrum

The /f noise at different biasing voltages was measured using a low-noise amplifier and a dynamic signal analyzer. The obtained low-frequency noise power spectrum is shown on Figure 7.

Images were focused on the carbon bolometer array using an AMTIR (Amorphous Material Transmitting Infrared) lens having F-number 1.5. (obtained from Newport) and a data acquisition system was used to individually access the bolometer pixels on a $12 * 11$ subset of the $32 * 32$ array. Also, small software application was written to extract thermal images. First, the resistance of each pixel is measured while no radiation is allowed to fall on the array. Then, the resistance of each pixel is measured again when radiation is incident on the array. Although the IR optics is far from being optimized, we managed to obtain IR images. Figure 8 (a) shows a soldering tip and Figure 8 (b) shows four resistors arranged in a rectangle and heated up to $202^{\circ} \mathrm{C}$ (the picture was spatially interpolated). The brightest pixel on Figure 7 (b) corresponds to a $-3.6 \%$ resistance change. In those experiments, the noise was mostly due to pick-up noise in the readout circuit outside the vacuum chamber.

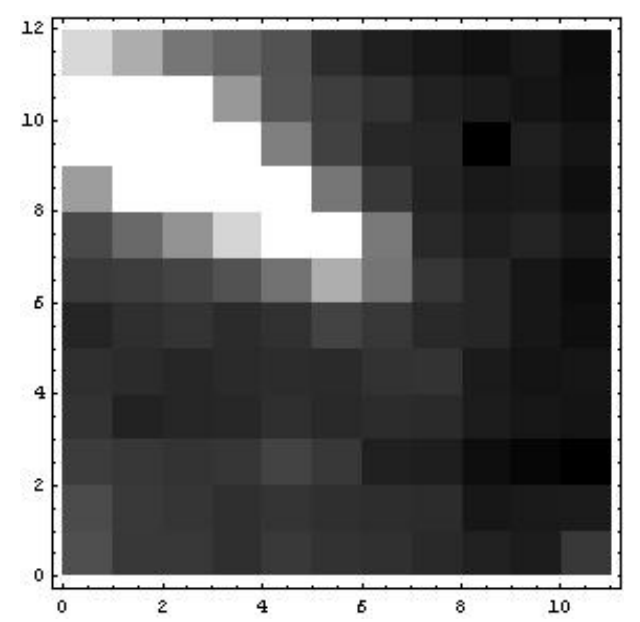

(a)

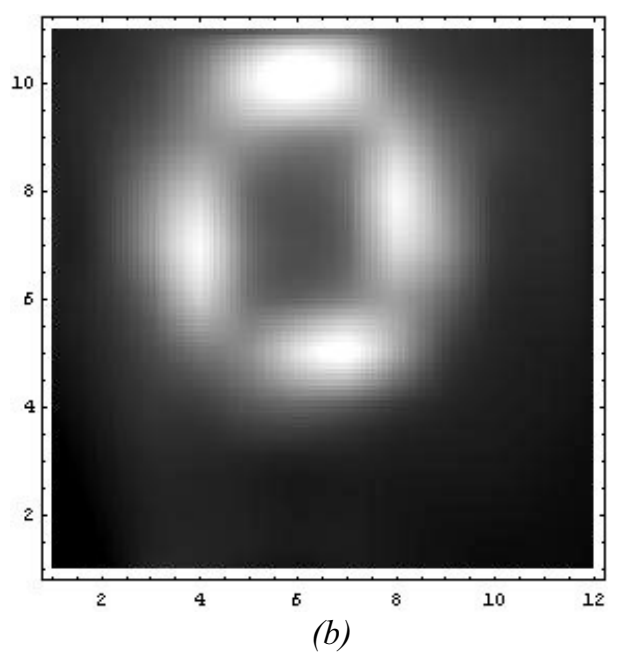

Figure 8:Obtained Thermal Images

\section{Noise Analysis}

Neglecting the temperature fluctuation noise and background noise, the voltage noise appearing in Equation 2 is given by

$$
V_{n}=\sqrt{V_{n, f}^{2}+V_{n, j}^{2}}
$$


where $V_{n, f}, V_{n, j}$ are the $1 / \mathrm{f}$ ("flicker") and thermal noise contributions respectively. The power spectral densities of these noise sources are given respectively by

$$
\begin{gathered}
S_{V, f}(f)=\frac{K V_{\text {bias }}^{2}}{f} \\
S_{V, j}(f)=4 k_{B} T R
\end{gathered}
$$

where $K$ is the $1 / \mathrm{f}$ noise factor depending on both the material and the volume of the device, $V_{\text {bias }}$ is the bias voltage, $k_{B}$ is Boltzmann's constant, $T$ is the temperature, and $R$ is the bolometer resistance. For a readout circuit that averages, or integrates, the signal over a period of time $\tau_{\text {pulse }}$, the total flicker and thermal noise contribution become [9]

$$
\begin{aligned}
& V_{n, f}^{2}=K V_{\text {bias }}^{2}\left[\frac{3}{2}-\gamma-\ln \left(2 \pi f_{1} \tau_{\text {pulse }}\right)\right] \\
& V_{n, j}^{2}=4 k T R\left(\frac{1}{2 \tau_{\text {pulse }}}-f_{1}\right)
\end{aligned}
$$

where $f_{1}$ is a frequency arbitrarily chosen to be the lowfrequency limit of both noise contributions and $\gamma \simeq 0.58$ is the Euler-Mascheroni constant. Using a $100 \mu$ s integration time, a bias of 5 Volts and a low-frequency limit to the noise of $1 \mathrm{mHz}$, the NETD is calculated to be $35 \mathrm{mK}$ (assuming the optical system has a transmission of $100 \%$ ). The temperature increasing during readout for such parameters is only $0.35 \mathrm{mK}$ due to the high resistance of the pixels.

Table 1 summarizes key parameters of the carbon microbolometer imager, compared to those of the VOx array reported in [8]. The reported $1 /$ f noise power spectral density for VOx bolometers is lower than our carbon bolometers: $2.5^{*} 10^{-12} / \mathrm{f} \mathrm{V}^{2} \mathrm{~Hz}^{-1}$ versus $1.6^{*} 10^{-10} / \mathrm{f} \mathrm{V}^{2} \mathrm{~Hz}^{-1}$ at $5 \mathrm{~V}$ bias. However, the carbon bolometer has higher responsivity and its higher resistance allows for a longer integration without

Table 1: Bolometer Array Parameters

\begin{tabular}{l|l|l} 
& $\begin{array}{l}\text { This } \\
\text { work }\end{array}$ & \multicolumn{1}{|c}{$[8]$} \\
\hline Pitch & $75 \mu \mathrm{m}$ & $50 \mu \mathrm{m}$ \\
\hline Pixel Area $\left(\mu \mathrm{m}^{2}\right)$ & $50 * 50$ & $35^{*} 35$ \\
\hline Fill Factor & $44 \%$ & $70 \%$ \\
\hline TCR $\left(\% . \mathrm{K}^{-1}\right)$ & -2.1 & -2.3 \\
\hline Average Pixel resistance & $3.8 \mathrm{M} \Omega$ & $20 \mathrm{k} \Omega$ \\
\hline Thermal conductance $\left(\mathrm{W} . \mathrm{K}^{-1)}\right.$ & $6.1^{*} 10^{-8}$ & $2^{*} 10^{-7}$ \\
\hline Thermal capacitance $\left(\mathrm{J} . \mathrm{K}^{-1}\right)$ & $1.9^{*} 10^{-9}$ & $3 * 10^{-9}$ \\
\hline Thermal time constant $(\mathrm{ms})$ & 31 & 15 \\
\hline Absorptivity & $55 \%$ & $80 \%$ \\
\hline DC Responsivity V.W $\mathrm{W}^{-1} @ 5 \mathrm{~V}$ & $9.5^{*} 10^{5}$ & $4.6^{*} 10^{5}$ \\
\hline Total noise $(\mu \mathrm{V})$ & 54 & $14(\mathrm{calc})$ \\
\hline Calculated NETD $(\mathrm{mK})$ & 35 & 39
\end{tabular}

excessive self-heating. Moreover, since the carbon used as the sensing material is obtained from pyrolysis, it is expected to be able to withstand higher temperature increase pulses than VOx. A $320 * 200$ carbon bolometer imager with an integration time of $100 \mu \mathrm{s}$. The NETD of the carbon bolometers is calculated to be slightly better than the VOx bolometers.

\section{CONCLUSIONS}

We demonstrated an uncooled microbolometer array based on a two-level self supporting carbon structure obtained through pyrolysis of parylene with process-tuned electrical and thermal properties. The responsivity, dynamic behavior and noise properties of this thermal imager show promising results, with a calculated NETD of $35 \mathrm{mK}$ under optimal conditions. Possible future directions include the development of a high fill factor surface-micromachined process, which could also help in increasing the absorptivity using a reflector.

\section{ACKNOWLEDGEMENTS}

The authors wish to thank Stacey Walker Boland and Scott Miserendino for their help with characterizing the material as well as Hsi-wen Lo for his help in building the testing setup. Trevor Roper is also acknowledged for equipment support.

\section{REFERENCES}

[1] P.E. Howard et al Proc.SPIE Vol. 3698131 (1999)

[2] Tissot JL, Infrared Physics \& Technology 43 (3-5) 223-228 Jun-Oct 2002

[3] J. Kim, X. Song, K. Kinoshita, M. Madou, and R. White, "Electrochemical studies of carbon films from pyrolyzed photoresist," J. Electrochem Soc, vol. 145, July 1998

[4] S. Ranaganathan, R. McCreery, S. M. Majji, and M. Madou, "Photoresist-derived carbon for microelectromechanical systems and electrochemical applications," Journal of The Electrochemical Society, vol. 147, no. 1, pp. 277-282, 2000

[5] S. Miserendino, S. Boland, and Y. C. Tai, "Material and electrochemical properties of pyrolyzed parylenec," in 206th Meeting of the Electrochemical Society, October 2004

[6] S. Konishi, M. Liger, T. Harder, and Y.C. Tai, "Parylene-Pyrolyzed Carbon for MEMS". The 17th IEEE International MEMS Conference (MEMS'04), Maastricht, Netherlands Jan 15-29 2004, p161.

[7] M. Liger, S. Konishi and Y.C. Tai, "Uncooled AllParylene Bolometer". The 17th IEEE International MEMS Conference (MEMS'04), Maastricht, Netherlands Jan 15-29 2004, p593.

[8] R.A. Wood, "Monolithic Silicon Microbolometer Arrays", Semiconductors and Semimetals, Vol 47, 1997.

[9] M. Liger "Uncooled Carbon Microbolometer Imager", PhD Thesis, California Institute of Technology, 2005. 